\title{
The Mechanism of Sesame Resistance Against Macrophomina Phaseolina Was Revealed via Comparison of Transcriptomes of Resistant and Susceptible Sesame Genotypes
}

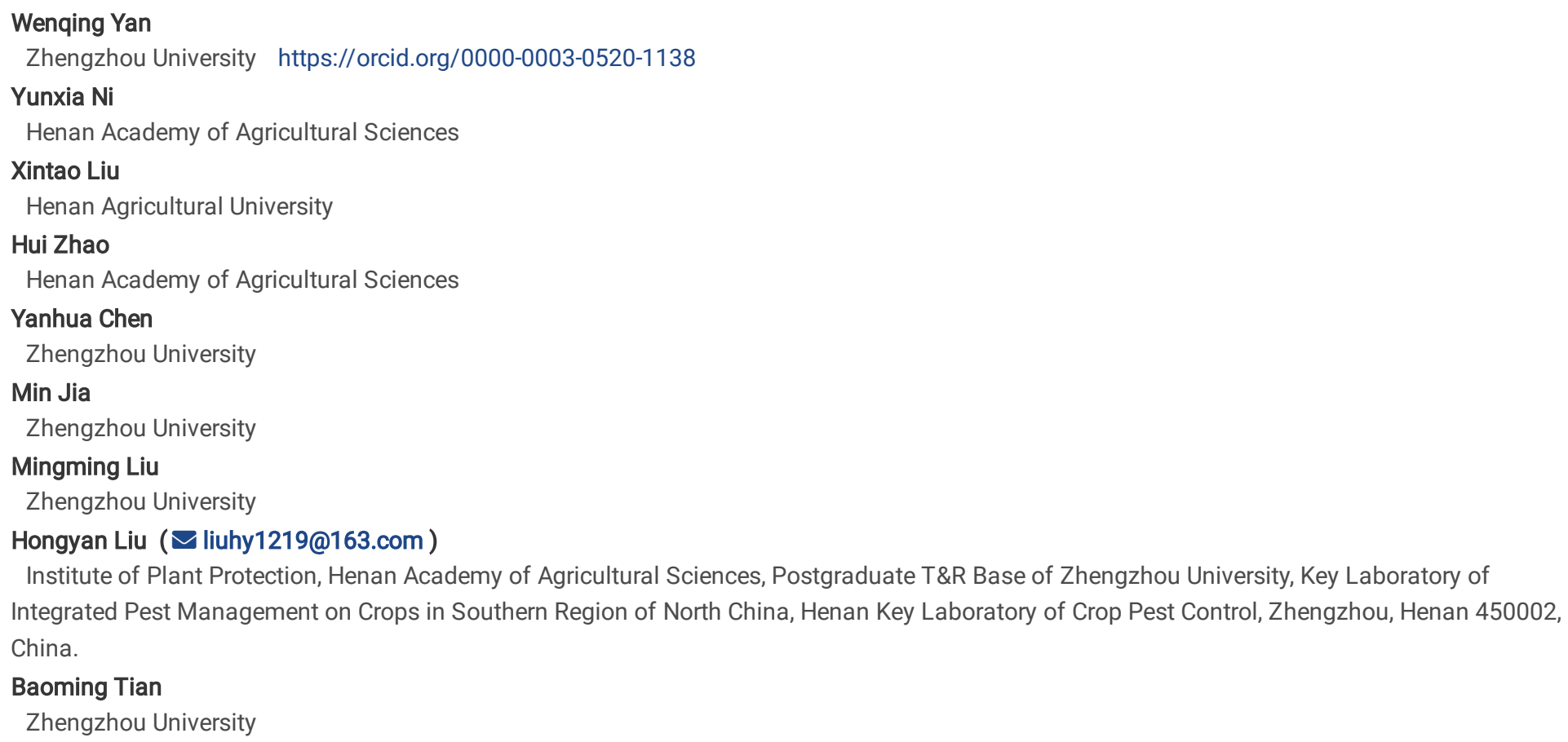




\section{Abstract}

Background: Sesame (sesamum indicum) charcoal rot, a destructive fungal disease caused by Macrophomina phaseolina (Tassi) Goid (MP), is a great threat to the yield and quality of sesame. However, there is a lack of information revealing gene-for-gene relationship between sesame and MP, and the molecular mechanism in the interaction is not yet clear. The aim of this study is to interpret the molecular mechanism of sesame resistance against MP in disease-resistant (DR) and disease-susceptible (DS) genotypes based on transcriptomes, which is the first report on the interaction between sesame and MP from the transcriptomes.

Results: A set of core genes that function in sesame disease resistance were revealed in our investigation by comparative transcriptomics, which preferentially associate with GO terms such as ribosome-related processes, fruit ripening, regulation of jasmonic acid mediated signaling pathway and cell-cell signaling. In addition, 52 potential resistance-related genes differentially and persistently expressed all through were obtained in sesame. Furthermore, we preliminarily studied the immune response mechanism of sesame against MP and found that auxin signal pathway shows negative regulation in the process. Also, the most highlighted pathway JA/ET signal transduction plays a negative regulatory role in sesame resistance against MP. Finally, a JAZ gene (LOC105168467) and an ERF transcription factor (LOC105171710) that may be related to disease resistance were screened.

Conclusions: In our investigation, the mechanism of resistance against MP from the transcriptomes were illustrated, which is a complex bioprocess involved in many phytohormones and disease resistance-related genes. The findings not only shed more light on mechanism of interaction between sesame and MP, but also help us identify key genes and transcription factors (TFs) associated with charcoal rot resistance in sesame.

\section{Background}

Being sessile, plants have evolved complex signaling systems and molecular mechanisms to cope with multifarious biotic stress and abiotic stress in a constantly changing environment [1]. In 2006, the concept of plant innate immunity was first put forward. The pattern-triggered immunity (PTI) and effector-triggered immunity (ETI) are two vital mechanisms in the long course of co-evolution of plant and pathogen interaction [2]. In spite of the fact that PTI is feeble, it is essential for plants and the first line of defense against pathogens [3]. PTI can be triggered by pathogen-associated molecular patterns (PAMPs), followed by thickening of cell wall, lignification of cell wall, production of phytoalexin and inducing expression of PR genes. Yet, some pathogens can restrain and break through the defense of PTI by secreting effectors into plants. However, plants have developed a reconnaissance mechanism to perceive and recognize these effectors, which leads to ETI. Both PTI and ETI are engaged with the early defense response of plants, they all perform comparative functions and early induction of defense signal transduction and downstream molecular network responses can also be observed from the physiological level, such as the burst of reactive oxygen species (ROS), the activation of mitogen activated protein kinases (MAPK) pathway and the amassing of callose [4]. The production of reactive oxygen intermediates, particularly the outbreak of superoxide anion radicals and the accumulation of hydrogen peroxide, is considered to be the early defense response of plants to external pathogens and the necessary autoimmune reaction process of plant [5]. ROS, including $\mathrm{O}^{2-}, \mathrm{H}_{2} \mathrm{O}_{2}$ and $\mathrm{HO}^{-}$, predominantly collects in chloroplasts and mitochondria, which can make oxidative damage to lipids, proteins, nucleic acids and photosynthetic devices. So as to reduce oxidative harm, plants produce different types of antioxidant enzymes such as superoxidase dismutase (SOD), catalase (CAT) and peroxidase (POD) to scavenge ROS to enhance their disease resistance. Simultaneously, the process of decomposing $\mathrm{H}_{2} \mathrm{O}_{2}$ by POD can likewise produce toxic substances to invasive microorganisms and inhibit the proliferation and diffusion of pathogens [6]. ROS is considered to be an essential signaling component in plant defense [7-8].

Basic helix-loop-helix (bHLH) proteins belong to TF superfamily and are widely distributed in eukaryotes. Members of the bHLH superfamily generally contain two highly conserved and functionally different domains: the N-terminal basic region that binds to DNA [9], which mainly recognizes E-box and G-box [10]; and the C-terminal HLH domain, which depends on the interaction of hydrophobic amino acids to form autodiploid or allodiploid of two HLH proteins and regulates the expression of downstream target genes [11-12]. bHLHs often cooperate with members of other TF families to regulate and induce the biosynthesis of an assortment of secondary metabolites like terpenoids, alkaloids, phenylpropanoid, anthocyanins and so on, which assume a significant job in regulating the interaction between plants and the environment [13-14]. Presently an everincreasing number of studies have demonstrated that bHLHs are related to biotic or abiotic stress reactions in various plants [15-17].

Sesame, a member of the Pedaliaceae family, is one of the most advantageous and nutritious oil crops with an oil substance of $50 \%-60 \%$ and a protein substance of $20 \%-30 \%$. Furthermore, it is wealthy in unsaturated fats (about $85 \%$ ) and natural antioxidants like sesamol, tocopherol and nutrient E [18-19]. These antioxidants have significant health-promoting effects, such as reducing cholesterol and hypertension, reducing the incidence of some cancers and neuroprotective effects against hypoxia. Subsequently, the worldwide demand for sesame has continuously expanded as of late. But sesame is vulnerable to a variety of pathogens in China, which are the leading causes for the low and unstable yield of sesame. In addition, the basic research on sesame is still scarce compared with other crops, which is also one of the reasons for low yield of sesame. It is necessary to study the basic genetics and molecular biology of sesame to improve the resistance of sesame to biotic stress.

Sesame charcoal rot generally occurs at the end of the flowering to the ripening stage of sesame, with the disease spots beginning to appear and spread from the root or stem under hot and dry weather or negative environmental stress. It is caused by a seed- and soil-borne fungus MP, which is 
highly contagious and can infect in excess of 500 species of plants. Charcoal rot can usually diminish sesame production of $10 \%-15 \%$, even over $80 \%$ in serious cases. Furthermore, it will likewise impact the quality of sesame by diminishing the oil substance of sesame seeds of $4.2 \%-16.2 \%$ [20]. Hence, revealing the resistance mechanism of sesame, screening the resistance genes in sesame and cultivating resistant varieties are helpful for us to lessen the loss of yield. Unfortunately, the genetic improvement of sesame is proceeding slowly due to the lack of the molecular mechanism and information regarding the gene-for-gene relationship in the interaction between sesame and MP. With the publication of sesame genomes [2122], the research on sesame has become more and more active. Hitherto, the investigation on the transcriptomes of the interaction between sesame and MP has not been published yet.

Consequently, this study preliminarily explored the response molecular mechanism of sesame resistance to MP by comparing and analyzing the transcriptome data of sesame resistant genotype DR and susceptible genotype DS inoculated with MP globally, which gave fundamental theoretical researches to the genetic improvement of sesame.

\section{Results}

Illumina sequencing and alignment to genome

Five time points post-inoculation ( $0 \mathrm{HPI}, 12 \mathrm{HPI}, 24 \mathrm{HPI}, 36 \mathrm{HPI}, 48 \mathrm{HPI})$ and their biological replicates of sesame were sequenced and 30 transcriptomes were obtained. The evaluation of sequencing quality indicated that the sequencing results of all samples were excellent, the base distribution was balanced and the mean Q value was about 36. More than 2.4 billion raw reads were generated from 30 libraries and then about 2.337 billion clean reads (clean ratio $>95.69 \%$ ) were obtained for subsequent analysis after removing adapter sequences, low-quality reads and rRNA sequences. On average, $95.89 \%$ of the reads can be mapped to the reference genome of sesame and most of them ( $88.28 \%$ ) can be mapped to the coding region (Additional file: Table S1, Fig. 1).

Check the relationship between samples through the pearson correlation coefficients between samples and principal component analyses (PCA) (Additional file: Figure S1). We selected out the samples with high correlation between biological replicates $\left(R^{2}>0.96\right)$. Furthermore, we can also see that the two samples of DS 0 h-2 and DR 48 h-3 are seriously outlier from PCA. Taken above, DS 0 h- 2 and DR 48 h-3 were excluded in the following analysis. PCA also showed that there was a great difference between control $(0 \mathrm{HPI})$ and treated groups, which indicated that the sesame transcriptomes regulated plenty of genes expressed under MP press, and there must be some resistance-related genes in the interaction between sesame and MP.

\section{Core gene sets in response to MP}

In order to apprehend the overall transcriptome changes of the interaction between sesame and MP in two genotypes, the genes with FPKM value greater than 0.1 were regarded as expressed genes. 22049,22114,21961,21712 and 22032 genes were detected in DS while

22514,22036,22032,22049 and 22100 genes were detected in DR at $0 \mathrm{HPI}, 12 \mathrm{HPI}, 24 \mathrm{HPI}, 36 \mathrm{HPI}, 48 \mathrm{HPI}$, respectively. The expression of 23042 and 23217 genes was also observed all time-points in DS and DR, respectively (Additional file: Figure S2A). There are 96.9\% (22761) of the genes expressed in both DS and DR, 1.2\% (281) of the genes were specifically expressed in DS and 1.9\% (456) in DR (Additional file: Figure S2B).

To investigate genes in response to MP in sesame, differentially expressed genes (DEGs) were identified under the standard of false discovery rate $($ FDR $)<0.01$ and $\mid \log 2$ Fold change| > 1. As shown in Fig. 2A, 3607, 3876, 3336 and 3359 DEGs were significantly up-regulated and $2839,3684,4329$ and 2956 DEGs down-regulated in DS, while 2304, 2410, 2485,2600 DEGs in DR were significantly up-regulated and 2803,2703,3091,2394 DEGs down-regulated at four time points post-inoculation, respectively. It follows that overall DEGs (4994-5576 DEGs) of DR was less than that of DS (6315-7665 DEGs) within 48 hours post-inoculation, and the number of DEGs responding to stress in DR was significantly less than that in DS at each time point. This indicated that the injury caused by MP in DR is much less than that in DS, which can change the transcriptome expression profile to a smaller extent and cope with the stress more leisurely.

Further overlap analysis of up- and down-regulated DEGs at four time points of DS and DR showed that 1977 and 1320 co-up-regulated genes and 1791 and 1357 co-down-regulated genes were identified in DS and DR respectively. To identify the core gene sets in response to MP, we compared the overlap DEGs between DS and DR and found that there are 867 up-regulated DEGs and 721 down-regulated DEGs overlapped between the two genotypes (Fig. 2B).

The enrichment of GO terms of core gene sets above was analyzed to study the potential function of genes in response to MP. The 867 up-regulated DEGs are mainly enriched in ribosome-related process, followed by thiamine pyrophosphate binding, maturation of LSU-rRNA from tricistronic rRNA transcript (SSU-rRNA, 5.8S rRNA, LSU-rRNA), fruit ripening, acylglycerol lipase activity and defense response to gram-negative bacterium (Fig. 3A). Likewise, the main term with the highest enrichment of 721 down-regulated DEGs are regulation of jasmonic acid mediated signaling pathway, UDPgalactosyltransferase activity, cell-cell signaling, response to freezing and regulation of secondary cell wall biogenesis (Fig. 3B, Additional file: Table S2).

DEGs up-regulated peculiarly in DR 
To study the functional specificity of disease resistance in DR, up-regulated DEGs observed peculiarly in DR compared with DS were explored along the time trend. At $12 \mathrm{HPI}, 733 \mathrm{DEGs}$ are enriched in the process of ribosome synthesis and assembly, maturation of SSU-rRNA from tricistronic rRNA transcript (SSU-rRNA, 5.8S rRNA, LSU-rRNA) and cytoplasmic translation (Fig. 4A), indicating that DR can respond more quickly than DS and prepare for translation of resistance-related proteins at the level of transcription and translation at the initial stage of stress. At $24 \mathrm{HPI}, 488 \mathrm{DEGs}$ are enriched best in GO terms, such as ligand-gated ion channel activity, cellular response to hypoxia, oxidoreductase activity, systemic acquired resistance, positive regulation of defense response and so on (Fig. 4B), which reveals that DR has made a series of responses to the infection stress, such as the production of peroxidase, activated the systemic acquired resistance process and defense response, illustrating that DR can arrange the defense system more quickly and effectively to resist MP. When the stress was more severe (36 HPI), a total of 737 DEGs are specifically upregulated in $\mathrm{DR}$, which are mainly enriched in ribosome-related processes, followed by phloem transport, nucleoside, nucleobase transport, nucleobase transmembrane transporter activity, cytoplasmic translation and hydrogen peroxide catabolic process (Fig. 4C). At 48 HPI, GO enrichment indicated that 750 DEGs involved in ribosome related pathways, cytoplasmic translation, RNA binding, beta-glucosidase activity, ligandgated ion channel activity and monoterpenoid biosynthetic process (Fig. 4D). It indicated that DR is continuously transcribing, translating and transporting disease resistance-related proteins and secondary metabolites such as monoterpenes to deal with the stress. All in all, the rapid stress responses and the activation of specific disease-related pathways of DR lead to its resistance.

DEGs between DS and DR

Moreover, DEGs were compared between DR and DS to filter the genes with high correlation with disease resistance in sesame. Under normal growth conditions (0 HPI), 1577 DEGs were observed between DR and DS (Fig. 5A). GO enrichment analysis showed that these genes were enriched in condensin complex, mitotic chromosome condensation, DNA primase activity, chromosome condensation, DNA unwinding involved in DNA replication, FMN reductase activity and response to anoxia (Additional file: Figure S3).

Then, based on overlapped analysis, a total of 52 genes that were significantly differentially expressed at all four time-points between the two genotypes were obtained (Fig. 5B, Additional file: Table S3). Then they were classified into 5 clusters exhibiting different functions (Fig. $5 \mathrm{C}$ ). These 52 genes are most enriched in GO terms such as response to abscisic acid, cell wall, hormone-mediated signaling pathway, response to hormone, cell-cell junction, defense response and signal transduction (Additional file: Figure S4), most of which are known to be associated with plant stress. This further confirms that these genes are crucial candidate genes inducing higher resistance in DR than DS. Among them, 20 of these genes exhibit significant differences in expression even under normal conditions between the two genotypes.

It is noteworthy that there were the most DEGs in two sesame genotypes at $36 \mathrm{HPI}$, as well as between the two genotypes (Fig. $2 \mathrm{~A}$, $5 \mathrm{~A}$ ). Furthermore, many DEGs in KEGG pathways related to biological stress are significantly enriched at $36 \mathrm{HPI}$ compared between DR and DS, including "plant hormone signal transduction" (35 DEGs), "plant-pathogen interaction" (15 DEGs), "brassinosteroid biosynthesis" (2 DEGs) and " diterpenoid biosynthesis " (12 DEGs). However, DEGs in these key signal pathways were not obvious at other time points, indicating that 36 HPI is an important period in the disease resistance of sesame (Additional file: Table S4).

DEGs involved in key pathways at $36 \mathrm{HPI}$

In the perspective of KEGG pathways, "Plant-pathogen interaction" and "Plant hormone signal transduction" are the key pathways in plant resistance. Therefore, the two main pathways in sesame were analyzed at the important time period of $36 \mathrm{HPI}$.

The genes involved in the "Plant-pathogen interaction" pathway were identified based on the KEGG pathway assignment. The results show that 48 and 72 DEGs were identified in DR and DS respectively, and most of these genes were down-regulated in both genotypes. In DR, the expression of PR1 (pathogenesis-related protein 1), HSP90 (heat shock protein 90 kDa beta), MAP2K1 (mitogen-activated protein kinase kinase 1 ) and RPM1 (disease resistance protein RPM1) increased, while the expression of WRKY22, WRKY29, WRKY33, Rboh (respiratory burst oxidase), FLS2 (LRR receptor-like serine/threonine-protein kinase FLS2) and CDPK (calcium-dependent protein kinase) decreased. In DS, the expression of BAK1 (brassinosteroid insensitive 1-associated receptor kinase 1), HSP90, MAP2K1 and Pti1 (pto-interacting protein 1) genes were up-regulated, while FLS2, MEKK1 (mitogen-activated protein kinase kinase kinase 1), NHO1 (glycerol kinase), Rboh, RPS2 (disease resistance protein RPS2), WRKY22, WRKY29 and WRKY33 genes were down-regulated. Furthermore, in the "Plant-pathogen interaction" pathway, up-regulated genes such as HSP90 were more involved in DR than in DS. For the genes that detected only in DS, the expression of BAK1 and Pti1 increased while the expression of MEKK1 and NHO1 decreased (Additional file: Table S6).

Similarly, in the "Plant hormone signal transduction" pathway, DEGs involved in DS (113) are more than that in DR (76). Most of these genes participate in auxin (AUX), abscisic acid (ABA) and ethylene (ET) biosynthesis. In addition, genes connected to CRE1 (Arabidopsis histidine kinase 2/3/4), B-ARR (two-component response regulator ARR-B family), SnRK2 (serine/threonine-protein kinase SRK2), EIN2 (ethylene-insensitive protein 2), BZR1_2 (brassinosteroid resistant 1/2) and BSK (BR-signalling kinase) specifically expressed in DS were all down-regulated while those related to BAK1 and BKI1 were all up-regulated (Additional file: Table S6).

In the " Plant-pathogen interaction" pathway, in comparison with DS, the expression levels of 2 CDPKs, 3 disease resistance protein RPM1, 1 LRR receptor-like serine/threonine-protein kinase FLS2, 2 CALM genes encoding calcium-binding protein and 1 Rboh gene in DR were significantly up-

Page $4 / 20$ 
regulated. These genes can induce PTI in plants by identifying $\mathrm{Ca}^{2+}$ signal and then activate hypersensitive response and cell wall reinforcment to prevent the spread of pathogens. The expression levels of other genes such as WRKY22 was significantly up-regulated, which can induce resistance by generating downstream defense gene. On the other hand, A total of 35 genes were differentially expressed in "Plant hormone signal transduction" pathway, with 11 DEGs up-regulated in connection with auxin, including those 2 IAA (auxin-responsive proteins), 2 AUX1 (auxin influx carriers), 2 auxin-responsive GH3 family, 1 ARF (auxin response factor) and 4 SAUR proteins. The other up-regulated genes were 2 genes encoding ethyleneresponsive transcription factor (ERF1) involved in ethylene response, 2 transcription factors TGA and 1 regulatory protein NPR1 related to SA biosynthesis, 2 cyclin D3 (CYCD3) involved in brassinosteroid pathway, 1 DELLA protein in gibberellin biosynthesis and 2 genes encoding twocomponent response regulator related to cytokinin synthesis pathway (Additional file: Table S7).

\section{TFs involved in sesame defense}

To research the major TFs of sesame in the interaction between sesame and MP, we investigated the expression of all genes involved in transcriptional regulation. A total of 3904 TFs were identified in the DEGs in sesame, which grouped into 49 gene families. In general, the number of transcription factors increased with the severity of stress (Additional file: Table S5). In DR, bHLH gene family, the most abundant and active TF family, is significantly higher than other transcription factor families, followed by ERF, MYB, NAC, WRKY, $\mathrm{C}_{2} \mathrm{H}_{2}$, LBD, GRAS, HD-ZIP, bZIP, ARF, MYB_related and other transcription factor families. Also, it could be seen that many DEGs were the members of TF families bHLH, MYB, ERF, NAC, WRKY, HD-ZIP, bZIP, GRAS, LBD, $\mathrm{C}_{2} \mathrm{H}_{2}$, ARF and HSF (Fig. 6). In this research, it was discovered that the bHLH transcription factor family was the most abundant transcription factor in the interaction between sesame and MP, indicating that bHLH proteins play a vital role in sesame charcoal rot resistance.

Real-time quantitative PCR

Five genes in two genotypes responded to MP were selected to confirm the RNA-seq results. LOC105160699 [LRR receptor-like serine/threonineprotein kinase (LRR-RTPK)] and LOC105169518 (LRR receptor-like serine/threonine-protein kinase) were associated with PTI while LOC105156719 (WRKY2) and LOC105157468 (disease resistance protein RPM1) were involved in ETI. Also, another gene LOC105167014 (PYL4) was related to plant hormone signal transduction. The results show that the data of real-time quantitative PCR and RNA-seq are consistent in the overall expression trend (Fig. 7).

\section{Discussion}

Comparative transcriptome analysis

Plants are generally subjected to a series of biotic and abiotic stresses, particularly pathogens stress, which seriously affect the growth and development of them. In our investigation, MP infection gradually changed the expression of sesame transcriptomes and demonstrated the most DEGs at $36 \mathrm{HPI}$, implying that it is the key period for sesame to resist the invasion of pathogens. In addition, we found that DS have more DEGs and TFs than DR regardless of the time point post-inoculation, indicating that susceptible genotypes were more likely to be interfered by MP at the transcriptional level, which may be due to the lack of corresponding mechanism in DS to adapt to MP stress. Different decisions made by DR and DS during pathogen infection led to their disparities in resistance. Based on GO enrichment analysis in all four time points, a great deal of DEGs engaged with ribosome-related procedures were collected in DR specifically, however these DEGs were not found in DS, which indicated that pathogen infection seems to specifically trigger adapted transcription responses in DR. Ribosomes are 'factories' that synthesize proteins at the cellular level and various mechanisms have been evolved to detect and react to environmental changes rapidly at transcriptional and translational levels in plants [23-24]. More DEGs involved in ribosome-related pathways in DR demonstrates that DR has a rapid and intenseresponse to MP stress with translational mechanisms activating synergistically with that of transcription, which is consistent with the consequences of Supriyo Chowdhury [25].

There are some DEGs constantly expressing in the two genotypes under MP stress, which represent the core genes mediating disease resistance against MP in sesame. It was discovered that many of these genes were PODs, and their expression increased significantly post-inoculation. Numerous studies have demonstrated that higher antioxidant enzyme activity helps to improve plant disease resistance [26-27]. POD participates in the defense against pathogens through its role in the detoxification of $\mathrm{H}_{2} \mathrm{O}_{2}$ and assumes an essential job in the process of disease resistance. When stressed by external pathogens, the enhancement of POD activity can increase the content of phenolic oxides to trigger hypersensitive responses, and afterward inhibit the proliferation and spread of pathogens [28]. There are also plenty of protein kinase genes and pathogenesisrelated genes in this core gene set. It is realized that a significant number of receptor protein kinases and pathogenesis-related protein can confer plant resistance against pathogens [29]. It is found that the PmDTM gene encoding receptor-like serine/threonine-protein kinase in wheat can improve the resistance of wheat to Blumeria graminis $f$. $s p$. tritici [30]. Similarly, CsWAKL08 gene encoding a wall-associated receptor-like kinase was found to regulate resistance against Xanthomonas citri subsp. citri positively via a mechanism of ROS control and JA signaling, which further features the significance of this kinase family in plant disease resistance [31]. ScPR10 was identified as a pathogenesis-related gene from sugarcane, which regulates plant resistance against Sporisorium scitamineum, Sorghum mosaic virus, salicylic acid and methyl jasmonate stresses positively [32]. Another important gene family identified in this core gene set is cytochrome P450, one of the largest gene families in the plant

Page 5/20 
genome. In wheat, the cytochrome P450 gene TaCYP72A was affirmed to be identified with the resistance to deoxynivalenol, which mediated the early resistance of wheat to F. graminearum [33]. Likewise, Guilin Wang et al. discovered that GbCYP86A1-1 gene in Gossypium barbadense plays a positive role in the resistance against Verticillium dahliae, it initiates the downstream immune pathways of disease resistance. For instance, GbCYP86A1-1 transgenic Arabidopsis significantly increased genes encoding the protein kinases, TFs and PRs, thereby increasing its resistance [34]. What's more, a few genes encoding laccase also reflected in this gene set. Yan Zhang et al. reported that GhLAC15 gene contained domains conserved by laccases enhances resistance against Verticillium dahliae by means of an increase of lignification and accumulation of arabinose and xylose [35], which indicates that laccase may have a significant relationship with the resistance of plants to pathogens.

Furthermore, the gene expression of DR and DS during stress was compared, and 52 genes were screened as differentially and continuously expressed, indicating that they may give DR higher tolerance against charcoal rot. Accordingly, further research may focus on these genes to identify their functions and find favorable genes with high disease resistance in sesame.

Vital KEGG pathways involved in sesame resistance against MP

KEGG enrichment analysis showed that there were plenty of DEGs involved in the two important pathways 'plant-pathogen interaction' and 'plant hormone signal transduction' at $36 \mathrm{HPI}$, which indicated that these two pathways also play an important role in the immune response of sesame. At present, both two pathways have been discussed and analyzed in detail in many investigations between plants and pathogens, such as the interaction between V.dahliae and sunflower [36] and the interaction between Penicillium oxalicum and orchidaceae [37], here we discuss and investigate the interaction between sesame and MP in detail.

"plant-pathogen interaction" pathway is generally considered to be one of the most important ways to initiate defense response during the interaction between plants and pathogens. According to Fig. 8, we can see that PR1,MAP2K1,RPM1 and HSP90 genes were up-regulated in DR while other genes were down-regulated or mixed-regulated, indicating that these genes may contribute to the resistance in DR. Interestingly, we found 7 HSP90 genes up-regulated in DR, significantly more than 4 in DS, and it is realized that many HSP90 genes have been confirmed to be associated with plant biotic stress [38-40]. All DEGs in DR above were involved in the process of hypersensitive response (HR), cell wall reinforcment, defense-related gene induction and phytoalexin accumulation in PTI and HR in ETI, proving these genes are important to the resistance against MP in sesame.

As key signaling molecules, phytohormones assume an essential function in signaling networks that regulate plant responses to various biological stresses [41], such as ZmAuxPR1 can respond to pathogen stress and participate in its resistance to Fusarium ear rot and Gibberella by a rapid reducing of its expression in maize [42]; $A B A$, which is the substrate of disease resistance gene Lr34 in wheat, can enhance its resistance to fungal pathogens [43];Cytokinin has also been proved that it can induce the expression of SNC1, a plant immune receptor gene in Arabidopsis thaliana, thereby improving its disease resistance [44]; In cucumber, T.longibrachiatum $\mathrm{H} 9$ can activate JA/ET and SA signaling pathways to enhance resistance to B.cinerea. [45]; Shaoqun Zhou et al. found that ethylene signal can regulate the resistance of maize to F.graminearum [46]; Gibberellin signaling also mediates plant sensitivity to Ralstonia solanacearum and Rhizoctonia solani via increasing gibberellin 3 content in tobacco. Similarly, activation of brassinosteroids and ethylene signaling pathways in rice can cooperatively regulate the resistance to Rhizoctonia solani. [47]; SA and JA have also been proved that they play a vital role in resistance against Fusarium spp. in wheat [48]. In our investigation, most DEGs related to biotic stress were enriched in the biosynthesis of AUX, ABA, ET, cytokinine and JA, indicating that complex networks of diverse plant hormones play a significant role in the interaction between sesame and MP (Fig. 9). Furthermore, many DEGs such as EIN3, JAZ (jasmonate ZIM domain-containing protein) and MYC2 involved in ET/JA pathway were down-regulated significantly, implying that JA/ET signal transduction plays a negative regulatory role in the sesame resistance against MP. Interestingly, we found that a JAZ gene (LOC105168467) and an ERF transcription factor (LOC105171710) were down-regulated in DR but up-regulated in DS, indicating that these two genes may also be related to the sesame resistance, which requires to study follow-up bio-function.

Defense-related TFs

It has been reported that plenty of TFs like bHLH [49-50], MYB [51-52], ERF [53-55], NAC [56-57], WRKY [58-60] were related to various plant resistance against pathogens. In our study, the bHLH transcription factor family was the most abundant transcription factor in the interaction between sesame and MP. It is known that many members of the bHLH family are related to abiotic stress resistance of plants, such as drought tolerance [61], cold tolerance [62] and salt tolerance [63]. However, their roles in plant biotic stress are rarely depicted. Until recently, Qun Cheng et al. revealed the role of a bHLH transcription factor GmPIB1 gene in soybean phytophthora root rot and found that GmPIB1 can directly bind to the promoter of the key enzyme GmSPOD1 gene that encodes ROS and inhibit its expression, reducing the production of ROS and enhancing the resistance of soybean to Phytophthora [49]. Yan S et al. discovered that cucumber transgenic plants with bHLH transcription factor CsIVP-RNAi had higher resistance to downy mildew and could accumulate higher level of SA. CsIVP can physically interact with CsNIMIN1, a negative regulatory factor in SA signal pathway, thus CsIVP is a significant regulatory factor in SA-mediated downy mildew resistance in cucumber [50]. Here, the current investigation also got the similar result that bHLH transcription factors play an important role in sesame resistance (Fig. 6).

\section{Conclusions}


To summarize, first of all, core disease resistance gene sets and 52 genes that may mediate the higher resistance of DR were obtained in sesame via comparative transcriptome analysis. In the process of MP stress, a group of core genes were activated to resist the infection of pathogens, including disease resistance-related protein kinase genes, pathogenesis-related genes, cytochrome P450 genes, peroxidase genes and so on. Then we further compared DEGs between DR and DS during the stress and found that 52 genes were differentially and persistently expressed, demonstrating that they may impart DR higher resistance against MP.

Then, we preliminarily investigated the immune response mechanism of sesame against MP and we can conclude that highlighted pathway JA/ET signal transduction plays a negative regulatory role in the process. This is the first report on the interaction between sesame and MP from the transcriptomes, which provides more viewpoints to understand the molecular mechanism of sesame against MP. Firstly, PTI and part of ETI in sesame were triggered in the infection. Secondly, auxin signal pathway shows negative regulation in sesame resistance against MP. Thirdly, SA signal transduction is also involved in sesame in response to MP, but its mechanism is complex and requires to be further revealed. Last but not least, two genes [a JAZ gene (LOC105168467) and an ERF transcription factor (LOC105171710)] may contribute to resistance against MP were likewise detected in plant hormone signal transduction pathway.

Finally, we found that bHLHs may play an important potential regulatory role under MP stress, for they were the most abundant and active transcription factor family in the process of MP infection. Findings above not only help us shed an insight to mechanism of interaction between sesame and MP, but also provide us important foundation for breeding for cultivars with high resistance.

\section{Methods}

Materials and stress treatment

Disease-susceptible genotype (Ji 9014) and disease-resistant genotype (Zhengzhi No.13) [64-65] used in this experiment were widely planted varieties and both of them were provided by Sesame Research Center, Henan Academy of Agricultural Sciences. Macrophomina phaseolina (MP), the pathogen of charcoal rot, was isolated and preserved by Biocontrol Lab, Institute of Plant Protection, Henan Academy of Agricultural Sciences.

Preparation of stroma mixed with MP: The MP strain stored in $25 \%$ glycerol at $-20^{\circ} \mathrm{C}$ was activated on PDA solid medium. After activation, MP was cultured in PDA solid medium and incubated in $30{ }^{\circ} \mathrm{C}$ incubator for 4 days. The PDA culture medium full of MP was divided into pieces width of about $0.5 \mathrm{~cm}$ with a sterilized toothpick, and then they were inoculated into the sterilized $200 \mathrm{~mL}$ liquid PD medium (each bottle was inoculated with half a plate of PDA). The medium was shaken and cultured for 5 days at the condition of $30^{\circ} \mathrm{C}$ and $200 \mathrm{r} / \mathrm{min}$. The mycelium suspension was obtained by breaking the culture medium full of mycelium with a tissue crusher. Then every $100 \mathrm{~mL}$ mycelium suspension was mixed with $100 \mathrm{~mL}$ sterilized water and sterilized stroma (nutritional soil: vermiculite $=3: 1) 200 \mathrm{~g}$.

The sesame seedlings were cultured in the growth bowl of stroma (soil: nutritional soil: vermiculite = 3:1:1), thinning them in 2 pairs of true leaf stage, leaving 3 seedlings in each pot. They were cultured in artificial climate box under the condition of $16 \mathrm{~h} \mathrm{light}\left(30^{\circ} \mathrm{C}\right)$ and $8 \mathrm{~h}$ darkness $\left(28^{\circ} \mathrm{C}\right)$. DS and DR were transplanted from the growth bowl to the stroma with mycelium carefully at three pairs of true leaves. The root tissues of DS and DR were collected as samples (three biological replicates) for RNA extraction during MP treatment (12 h, $24 \mathrm{~h}, 36 \mathrm{~h}, 48 \mathrm{~h})$ and before treatment ( $0 \mathrm{~h}$ ).

mRNA Library construction and sequencing

Total RNA extracted with TransZol Up Plus RNA Kit (Cat\#ER501-01, Trans) was qualified by Agilent Bioanalyzer 2100 (Agilent technologies, Santa Clara, CA, US) electrophoresis and purified with RNA Clean XP Kit (Cat A63987, Beckman Coulter, Inc.Kraemer Boulevard Brea, CA, USA) and RNase-

Free DNase Set (Cat\#79254, QIAGEN, GmBH, Germany). The quality of total RNA was checked by NanoDrop ND-2000 spectrophotometer and Agilent Bioanalyzer 2100 (Agilent technologies, Santa Clara, CA, US), and the RNA with high quality inspection could be sequenced later. According to the experimental operation instructions, the purified total RNA was subjected to mRNA separation, fragmentation, first-strand cDNA synthesis, secondstrand cDNA synthesis, terminal repair, 3'- terminal addition of A, adapters' junction, enrichment and other steps to complete the construction of cDNA library. After the construction of the library, Qubit @2.0 Fluorometer was used to detect the concentration, and Agilent4200 was used to detect the size of the library.

Sequencing: according to the corresponding process shown by cBot User Guide, Cluster generation and first-direction sequencing primer hybridization were completed on the cBot equipped with Illumina sequencer, and paired-end sequencing was carried out. The sequencing process and real-time data analysis are controlled by data collection software provided by Illumina.

\section{Transcriptome assembly}

Before downstream analysis, unqualified reads with low quality, primer sequence and low terminal quality should be removed. Seqtk package is used to filter the raw reads to get the clean reads so that reads can be used for subsequent data analysis. After filtering, the clean reads were mapped to sesame genome with HISAT2 (version:2.0.4) [66]. The data generated by Mapping is a BAM file. 
The sesame reference genome is S_indicum_v1.0 [22], which can be downloaded from:

ftp://ftp.ncbi.nlm.nih.gov/genomes/all/GCF/000/512/975/GCF_000512975.1_S_indicum_v1.0/GCF_000512975.1_S_indicum_v1.0_genomic.fna.gz.

Analysis of DEGs

Standardize gene expression by transforming reads into FPKM(fragments per kilobase of exon model per million mapped reads)to calculate gene expression level of each sample [67]. We first count the fragments number of each gene after Hisat2 alignment by Stringtie (version:1.3.0) [68-69], then normalize it by TMM (trimmed mean of M values) method [70], and finally use perl script to calculate the FPKM value of each gene.

Differential genes between samples were analyzed by edgeR package [71], and the p-value was corrected by multiple hypothesis testing. The threshold of $p$-value was determined by controlling False Discovery Rate (FDR), and the corrected p-value was q-value [72-73]. The differently expressed genes (DEG) were detected based on the parameters: $\log _{2} \mid$ Fold change| $>=1$ and q-value $<=0.05$.

Functional annotation and TFs prediction

DEGs were compared with the NCBI non-redundant (NR) database and were functionally annotated into databases GO and KEGG by KAAS.

To identify the transcription factors (TFs) in sesame DEGs, the online website plantTFDB [74] (http://planttfdb.cbi.pku.edu.cn/index.php?sp=Sin) was used.

qRT-PCR

Relative expression levels of 5 genes in DS and DR quantified by CFX $384^{\text {TM }}$ real-time System made in Singapore and ChamQ Universal SYBR qPCR Master Mix were calculated with the $2^{-\Delta \Delta C t}$ method. Each sample had 3 replicates. The primers of qPCR designed and synthesized in Sangon Biotech were shown as Additional file: Table S8. The relative expression levels of 5 genes were normalized to that of SiUBQ5 gene [75].

\section{Statistical analysis}

All data in this study are mean values of three biological replicates. FPKM value was used to depicted gene expression abundance.

\section{Abbreviations}

MP

Macrophomina phaseolina; DR:Disease-resistant; DS:Disease-susceptible; TF:Transcription factor; PTI:Pattern-triggered immunity; ETI:Effectortriggered immunity; PAMP:Pathogen-associated molecular pattern; ROS:Reactive oxygen species; MAPK:Mitogen activated protein kinase; SOD:Superoxidase dismutase; CAT:Catalase; POD:Peroxidase; bHLH:Basic helix-loop-helix; HPI:Hours post-inoculation; PCA:Principal component analyses; DEG:Differentially expressed genes; FDR:False discovery rate; PR1:Pathogenesis-related protein 1; HSP90:Heat shock protein 90 kDa beta; MAP2K1:Mitogen-activated protein kinase kinase 1; RPM1:Disease resistance protein RPM1; Rboh:Respiratory burst oxidase; FLS2:LRR receptor-like serine/threonine-protein kinase FLS2; CDPK:Calcium-dependent protein kinase; BAK1:Brassinosteroid insensitive 1-associated receptor kinase 1; Pti1:Pto-interacting protein 1; MEKK1:Mitogen-activated protein kinase kinase kinase 1; NH01:Glycerol kinase; RPS2:Disease resistance protein RPS2; AUX:Auxin; ABA:Abscisic acid; JA:Jasmonic acid; SA:Salicylic acid; ET:Ethylene; CRE1:Arabidopsis histidine kinase 2/3/4; B-ARR:Twocomponent response regulator ARR-B family; SnRK2:Serine/threonine-protein kinase SRK2; EIN2:Ethylene-insensitive protein 2;

BZR1_2:Brassinosteroid resistant 1/2; BSK:BR-signalling kinase; CALM:Calmodulin; GH3:Glycoside hydrolase; ARF:Auxin response factor; ERF1:Ethylene-responsive transcription factor 1; CYCD3:Cyclin D3; HR:Hypersensitive response; JAZ:Jasmonate ZIM domain-containing protein; FPKM:Fragments per kilobase of exon model per million mapped reads.

\section{Declarations}

\section{Ethics approval and consent to participate}

Not applicable

\section{Consent for publication}

Not applicable

\section{Availability of data and materials}


The sequence data generated in this study is available from the corresponding author on reasonable request. All data generated or analysed during this study are included in this published article and its additional files.

\section{Competing interests}

The authors declare that they have no competing interests.

\section{Funding}

This work was supported by the earmarked fund for China Agriculture Research System (CARS-14). The funding body had no role in the design of the study and collection, analysis, and interpretation of data and in writing the manuscript should be declared.

\section{Authors' contributions}

$\mathrm{HL}, \mathrm{BT}, \mathrm{YN}, \mathrm{XL}$ and $\mathrm{HZ}$ assisted in experiment conducting. $\mathrm{YC}$ and $\mathrm{MJ}$ prepared the plant materials. ML assisted in experiment and WY performed experiment, data analysis and manuscript writing. All authors read and approved the final manuscript.

\section{Acknowledgements}

We are grateful to Sesame Research Center, Henan Academy of Agricultural Sciences for sesame varieties. We also thank Chun Li for providing technical assistance.

\section{References}

[1] Bacete L, Mélida H, Miedes E, Molina A. Plant cell wall-mediated immunity: cell wall changes trigger disease resistance responses. Plant J. 2018;93(4):614-636.

[2] Jones JD, Dangl JL. The plant immune system. Nature. 2006;444(7117):323-329.

[3] Peng HC, Kaloshian I. The tomato leucine-rich repeat receptor-like kinases SISERK3A and SISERK3B have overlapping functions in bacterial and nematode innate immunity. PLoS One. 2014;9(3): e93302.

[4] Nicaise V, Roux M, Zipfel C. Recent advances in PAMP-triggered immunity against bacteria: pattern recognition receptors watch over and raise the alarm. Plant Physiol. 2009;150(4):1638-1647.

[5] Kim DS, Hwang BK. An important role of the pepper phenylalanine ammonia-lyase gene (PAL1) in salicylic acid-dependent signalling of the defence response to microbial pathogens. J Exp Bot. 2014;65(9):2295-2306.

[6] Zhang SS, Hu L, Liu ZL, et al. Relationship between the disease defense-related enzymes and the disease resistance of plants[J]. Anhui Agricultural Science Bulletin. 2006;(13):48-49.

[7] Van Breusegem F, Dat JF. Reactive oxygen species in plant cell death. Plant Physiol. 2006;141(2):384-90.

[8] Suzuki N, Koussevitzky S, Mittler R, Miller G. ROS and redox signalling in the response of plants to abiotic stress. Plant Cell Environ. 2012;35(2):259-270.

[9] Murre C, McCaw PS, Vaessin H, et al. Interactions between heterologous helix-loop-helix proteins generate complexes that bind specifically to a common DNA sequence. Cell. 1989;58(3):537-544.

[10] Jones S. An overview of the basic helix-loop-helix proteins. Genome Biol. 2004;5(6):226.

[11] Nesi N, Debeaujon I, Jond C, Pelletier G, Caboche M, Lepiniec L. The TT8 gene encodes a basic helix-loop-helix domain protein required for expression of DFR and BAN genes in Arabidopsis siliques. Plant Cell. 2000;12(10):1863-1878.

[12] Martínez-García JF, Huq E, Quail PH. Direct targeting of light signals to a promoter element-bound transcription factor. Science. 2000;288(5467):859-863.

[13] Chen L, Hu B, Qin Y, Hu G, Zhao J. Advance of the negative regulation of anthocyanin biosynthesis by MYB transcription factors. Plant Physiol Biochem. 2019; 136:178-187. 
[14] Zhou M, Memelink J. Jasmonate-responsive transcription factors regulating plant secondary metabolism. Biotechnol Adv. 2016;34(4):441-449.

[15] Abe H, Urao T, Ito T, Seki M, Shinozaki K, Yamaguchi-Shinozaki K. Arabidopsis AtMYC2 (bHLH) and AtMYB2 (MYB) function as transcriptional activators in abscisic acid signaling. Plant Cell. 2003;15(1):63-78.

[16] Chinnusamy V, Ohta M, Kanrar S, et al. ICE1: a regulator of cold-induced transcriptome and freezing tolerance in Arabidopsis. Genes Dev. 2003;17(8):1043-1054.

[17] Kavas M, Baloğlu MC, Atabay ES, Ziplar UT, Daşgan HY, Ünver T. Genome-wide characterization and expression analysis of common bean bHLH transcription factors in response to excess salt concentration. Mol Genet Genomics. 2016;291(1):129-143.

[18] Makinde FM, Akinoso R. Comparison between the nutritional quality of flour obtained from raw, roasted and fermented sesame (Sesamum indicum L.) seed grown in Nigeria. Acta Sci Pol Technol Aliment. 2014; 13:309-19.

[19] Nagendra Prasad M, Sanjay K, Prasad D, Vijay N, Kothari R, \& Nanjunda Swamy S. A review on nutritional and nutraceutical properties of sesame. J Nutr Food Sci. 2012; 2(2), 1-6.

[20] Wang L, Zhang Y, Zhu X, et al. Development of an SSR-based genetic map in sesame and identification of quantitative trait loci associated with charcoal rot resistance. Sci Rep. 2017;7(1):8349.

[21] Zhang H, Miao H, Wang L, et al. Genome sequencing of the important oilseed crop Sesamum indicum L. Genome Biol. 2013;14(1):401.

[22] Wang L, Yu S, Tong C, Zhao Y, Liu Y, Song C, Zhang Y, Zhang X, Wang Y, Hua W, Li D, Li D, Li F, Yu J, Xu C, Han X, Huang S, Tai S, Wang J, Xu X, Li Y, Liu S, Varshney RK, Wang J, Zhang X. Genome sequencing of the high oil crop sesame provides insight into oil biosynthesis. Genome Biol. 2014;15(2):R39.

[23] Dossa K, Li D, Wang L, et al. Transcriptomic, biochemical and physio-anatomical investigations shed more light on responses to drought stress in two contrasting sesame genotypes. Sci Rep. 2017;7(1):8755.

[24] Lei L, Shi J, Chen J, et al. Ribosome profiling reveals dynamic translational landscape in maize seedlings under drought stress. Plant J. 2015;84(6):1206-1218.

[25] Chowdhury S, Basu A, Kundu S. Biotrophy-necrotrophy switch in pathogen evoke differential response in resistant and susceptible sesame involving multiple signaling pathways at different phases. Sci Rep. 2017;7(1):17251.

[26] Narayan OP, Verma N, Singh AK, et al. Antioxidant enzymes in chickpea colonized by Piriformospora indica participate in defense against the pathogen Botrytis cinerea. Sci Rep. 2017;7(1):13553.

[27] Wu W, Wan X, Shah F, Fahad S, Huang J. The role of antioxidant enzymes in adaptive responses to sheath blight infestation under different fertilization rates and hill densities. Scientific World Journal. 2014; 2014:502134.

[28] Wang AL, Deng WQ, Zhu HQ, et al. On the change of enzyme activities of cucumber infected by Pseudoperon ospora [J]. Journal of Changjiang Vegetables. 2008;(10):52-54.

[29] Wan J, Zhang XC, Neece D, et al. A LysM receptor-like kinase plays a critical role in chitin signaling and fungal resistance in Arabidopsis. Plant Cell. 2008;20(2):471-481.

[30] Lu N, Lu M, Liu P, et al. Fine Mapping a Broad-Spectrum Powdery Mildew Resistance Gene in Chinese Landrace Datoumai, PmDTM, and Its Relationship with Pm24. Plant Dis. 2020;104(6):1709-1714.

[31] Li Q, Hu A, Qi J, et al. CsWAKL08, a pathogen-induced wall-associated receptor-like kinase in sweet orange, confers resistance to citrus bacterial canker via ROS control and JA signaling. Hortic Res. 2020; 7:42.

[32] Peng Q, Su Y, Ling H, et al. A sugarcane pathogenesis-related protein, ScPR10, plays a positive role in defense responses under Sporisorium scitamineum, SrMV, SA, and MeJA stresses. Plant Cell Rep. 2017;36(9):1427-1440.

[33] Gunupuru LR, Arunachalam C, Malla KB, et al. A wheat cytochrome P450 enhances both resistance to deoxynivalenol and grain yield. PLoS One. 2018;13(10): e0204992.

[34] Wang G, Xu J, Li L, et al. GbCYP86A1-1 from Gossypium barbadense positively regulates defence against Verticillium dahliae by cell wall modification and activation of immune pathways. Plant Biotechnol J. 2020;18(1):222-238. 
[35] Zhang Y, Wu L, Wang X, Chen B, Zhao J, Cui J, Li Z, Yang J, Wu L, Wu J, Zhang G, Ma Z. The cotton laccase gene GhLAC15 enhances Verticillium wilt resistance via an increase in defence-induced lignification and lignin components in the cell walls of plants. Mol Plant Pathol. 2019;20(3):309322.

[36] Guo S, Zuo Y, Zhang Y, Wu C, Su W, Jin W, Yu H, An Y, Li Q. Large-scale transcriptome comparison of sunflower genes responsive to Verticillium dahliae. BMC Genomics. 2017;18(1):42.

[37] Wang Y, Gao Y, Zang P, Xu Y. Transcriptome analysis reveals underlying immune response mechanism of fungal (Penicillium oxalicum) disease in Gastrodia elata BI. f. glauca S. chow (Orchidaceae). BMC Plant Biol. 2020;20(1):445.

[38] Ito M., Ohnishi K., Hikichi Y., Kiba A. Molecular Chaperons and Co-Chaperons, Hsp90, RAR1, and SGT1 Negatively Regulate Bacterial wilt Disease Caused by Ralstonia solanacearum in Nicotiana benthamiana. Plant Signal. Behav. 2014;10: e970410.

[39] Bhattarai KK, Li Q, Liu Y, Dinesh-Kumar SP, Kaloshian I. The MI-1-mediated pest resistance requires Hsp90 and Sgt1. Plant Physiol. 2007;144(1):312-23.

[40] Lourenço-Tessutti IT, Souza Junior JD, Martins-de-Sa D, Viana AA, Carneiro RM, Togawa RC, de Almeida-Engler J, Batista JA, Silva MC, Fragoso RR, Grossi-de-Sa MF. Knock-down of heat-shock protein 90 and isocitrate lyase gene expression reduced root-knot nematode reproduction.

Phytopathology. 2015;105(5):628-37.

[41] Bari R, Jones JD. Role of plant hormones in plant defence responses. Plant Mol Biol. 2009;69(4):473-88.

[42] Ye J, Zhong T, Zhang D, Ma C, Wang L, Yao L, Zhang Q, Zhu M, Xu M. The Auxin-Regulated Protein ZmAuxRP1 Coordinates the Balance between Root Growth and Stalk Rot Disease Resistance in Maize. Mol Plant. 2019;12(3):360-373.

[43] Krattinger SG, Kang J, Bräunlich S, Boni R, Chauhan H, Selter LL, Robinson MD, Schmid MW, Wiederhold E, Hensel G, Kumlehn J, Sucher J, Martinoia E, Keller B. Abscisic acid is a substrate of the ABC transporter encoded by the durable wheat disease resistance gene Lr34. New Phytol. 2019;223(2):853-866.

[44] Wang S, Wang S, Sun Q, Yang L, Zhu Y, Yuan Y, Hua J. A Role of Cytokinin Transporter in Arabidopsis Immunity. Mol Plant Microbe Interact. 2017;30(4):325-333.

[45] Yuan M, Huang Y, Ge W, Jia Z, Song S, Zhang L, Huang Y. Involvement of jasmonic acid, ethylene and salicylic acid signaling pathways behind the systemic resistance induced by Trichoderma longibrachiatum H9 in cucumber. BMC Genomics. 2019;20(1):144.

[46] Zhou S, Zhang YK, Kremling KA, Ding Y, Bennett JS, Bae JS, Kim DK, Ackerman HH, Kolomiets MV, Schmelz EA, Schroeder FC, Buckler ES, Jander G. Ethylene signaling regulates natural variation in the abundance of antifungal acetylated diferuloylsucroses and Fusarium graminearum resistance in maize seedling roots. New Phytol. 2019;221(4):2096-2111.

[47] Yuan P, Zhang C, Wang ZY, Zhu XF, Xuan YH. RAVL1 Activates Brassinosteroids and Ethylene Signaling to Modulate Response to Sheath Blight Disease in Rice. Phytopathology. 2018;108(9):1104-1113.

[48] Wang L, Li Q, Liu Z, Surendra A, Pan Y, Li Y, Zaharia LI, Ouellet T, Fobert PR. Integrated transcriptome and hormone profiling highlight the role of multiple phytohormone pathways in wheat resistance against fusarium head blight. PLoS One. 2018;13(11): e0207036.

[49] Cheng Q, Dong L, Gao T, et al. The bHLH transcription factor GmPIB1 facilitates resistance to Phytophthora sojae in Glycine max. J Exp Bot. 2018;69(10):2527-2541.

[50] Yan S, Ning K, Wang Z, et al. CsIVP functions in vasculature development and downy mildew resistance in cucumber. PLoS Biol. 2020;18(3): e3000671.

[51] Zhang YL, Zhang CL, Wang GL, Wang YX, Qi CH, Zhao Q, You CX, Li YY, Hao YJ. The R2R3 MYB transcription factor MdMYB30 modulates plant resistance against pathogens by regulating cuticular wax biosynthesis. BMC Plant Biol. 2019;19(1):362.

[52] Cui J, Jiang N, Zhou X, Hou X, Yang G, Meng J, Luan Y. Tomato MYB49 enhances resistance to Phytophthora infestans and tolerance to water deficit and salt stress. Planta. 2018;248(6):1487-1503.

[53] Tezuka D, Kawamata A, Kato H, Saburi W, Mori H, Imai R. The rice ethylene response factor OsERF83 positively regulates disease resistance to Magnaporthe oryzae. Plant Physiol Biochem. 2019;135:263-271.

[54] Liu J, Wang Y, Zhao G, Zhao J, Du H, He X, Zhang H. A novel Gossypium barbadense ERF transcription factor, GbERFb, regulation host response and resistance to Verticillium dahliae in tobacco. Physiol Mol Biol Plants. 2017;23(1):125-134. 
[55] Sun X, Yu G, Li J, Liu J, Wang X, Zhu G, Zhang X, Pan H. AcERF2, an ethylene-responsive factor of Atriplex canescens, positively modulates osmotic and disease resistance in Arabidopsis thaliana. Plant Sci. 2018;274:32-43.

[56] Liu Q, Yan S, Huang W, Yang J, Dong J, Zhang S, Zhao J, Yang T, Mao X, Zhu X, Liu B. NAC transcription factor ONAC066 positively regulates disease resistance by suppressing the ABA signaling pathway in rice. Plant Mol Biol. 2018;98(4-5):289-302.

[57] Wang B, Wei J, Song N, Wang N, Zhao J, Kang Z. A novel wheat NAC transcription factor, TaNAC30, negatively regulates resistance of wheat to stripe rust. J Integr Plant Biol. 2018; 60(5):432-443.

[58] Duan Y, Jiang Y, Ye S, Karim A, Ling Z, He Y, Yang S, Luo K. PtrWRKY73, a salicylic acid-inducible poplar WRKY transcription factor, is involved in disease resistance in Arabidopsis thaliana. Plant Cell Rep. 2015;34(5):831-41.

[59] Gao YF, Liu JK, Yang FM, Zhang GY, Wang D, Zhang L, Ou YB, Yao YA. The WRKY transcription factor WRKY8 promotes resistance to pathogen infection and mediates drought and salt stress tolerance in Solanum lycopersicum. Physiol Plant. 2020;168(1):98-117.

[60] Zou L, Yang F, Ma Y, Wu Q, Yi K, Zhang D. Transcription factor WRKY30 mediates resistance to Cucumber mosaic virus in Arabidopsis. Biochem Biophys Res Commun. 2019;517(1):118-124.

[61] Liu W, Tai H, Li S, et al. bHLH122 is important for drought and osmotic stress resistance in Arabidopsis and in the repression of ABA catabolism. New Phytol. 2014;201(4):1192-1204.

[62] Xu W, Zhang N, Jiao Y, Li R, Xiao D, Wang Z. The grapevine basic helix-loop-helix (bHLH) transcription factor positively modulates CBF-pathway and confers tolerance to cold-stress in Arabidopsis. Mol Biol Rep. 2014;41(8):5329-5342.

[63] Zhou J, Li F, Wang JL, Ma Y, Chong K, Xu YY. Basic helix-loop-helix transcription factor from wild rice (OrbHLH2) improves tolerance to salt- and osmotic stress in Arabidopsis. J Plant Physiol. 2009;166(12):1296-1306.

[64] Zhao H, Miao HM, Gao HT, Ni YX, Wei LB, Liu HY. Evaluation and Identification of Sesame Germplasm Resistance to Macrophomina phaseolina [J]. Journal of Henan Agricultural Sciences, 2012;41(11):82-87.

[65] Chen YH. Identification of resistance of sesame varieties to stem rot and functional analysis of SiPYL4 and SiTLP genes [D]. Zhengzhou University, 2019.

[66] Kim D, Langmead B, Salzberg SL. HISAT: a fast spliced aligner with low memory requirements. Nat Methods. 2015;12(4):357-60.

[67] Mortazavi A, Williams BA, McCue K, Schaeffer L, Wold B. Mapping and quantifying mammalian transcriptomes by RNA-Seq. Nat Methods. $2008 ; 5(7): 621-8$.

[68] Pertea M, Pertea GM, Antonescu CM, Chang TC, Mendell JT, Salzberg SL. StringTie enables improved reconstruction of a transcriptome from RNA-seq reads. Nat Biotechnol. 2015;33(3):290-5.

[69] Pertea M, Kim D, Pertea GM, Leek JT, Salzberg SL. Transcript-level expression analysis of RNA-seq experiments with HISAT, StringTie and Ballgown. Nat Protoc. 2016;11(9):1650-67.

[70] Robinson MD, Oshlack A. A scaling normalization method for differential expression analysis of RNA-seq data. Genome Biol. $2010 ; 11(3): R 25$.

[71] Robinson MD, McCarthy DJ, Smyth GK. edgeR: a Bioconductor package for differential expression analysis of digital gene expression data. Bioinformatics. 2010;26(1):139-40.

[72] Benjamini Y, Hochberg Y. Controlling the false discovery rate: a practical and powerful approach to multiple testing. J Roy Statist Soc Ser B. 1995; 57:289-300.

[73] Benjamini Y, Yekutieli D. The control of the false discovery rate in multiple testing under dependency. Ann Statist. 2001; 29:1165-1188.

[74] Jin J, Tian F, Yang DC, Meng YQ, Kong L, Luo J, Gao G. PlantTFDB 4.0: toward a central hub for transcription factors and regulatory interactions in plants. Nucleic Acids Res. 2017;45(D1):D1040-D1045.

[75] Liu LM, Liu HY, Tian BM. Selection of Reference Genes from Sesame Infected by Macrophomina phaseo-lina [J]. Acta Agronomica Sinica. 2012;38(03):471-478.

\section{Figures}




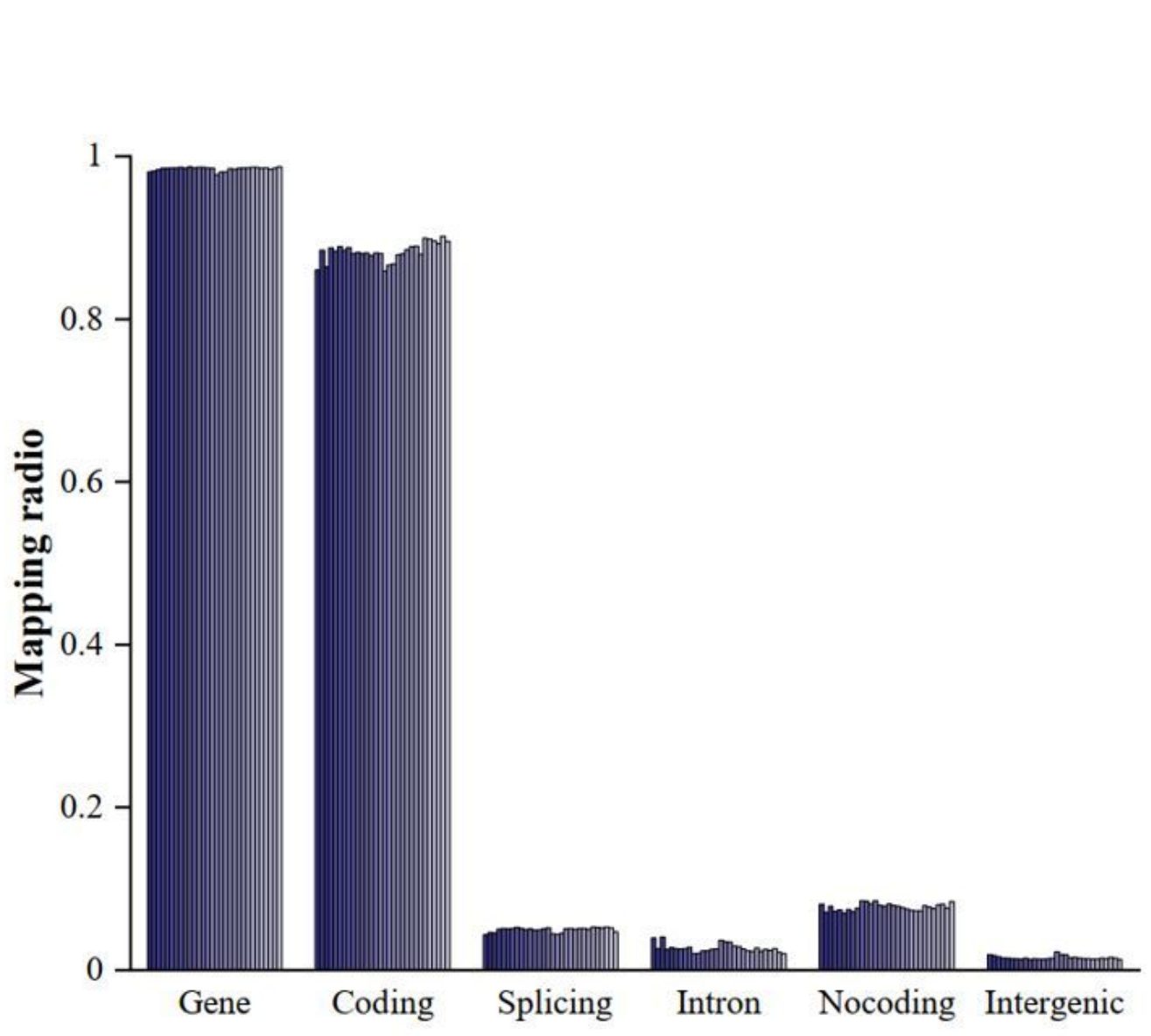

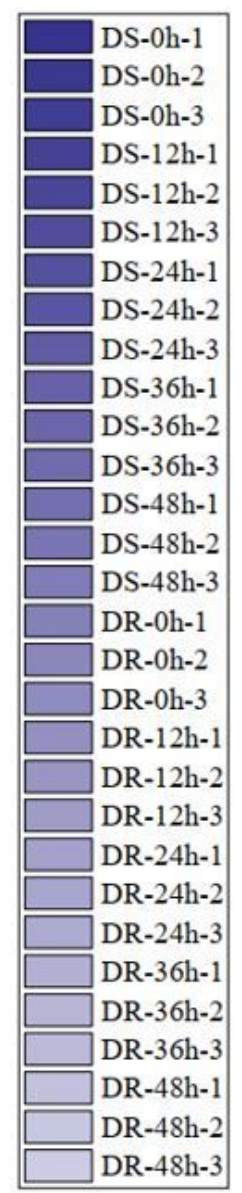

Figure 1

Mapping region distribution of 30 samples. 
$\mathbf{A}$

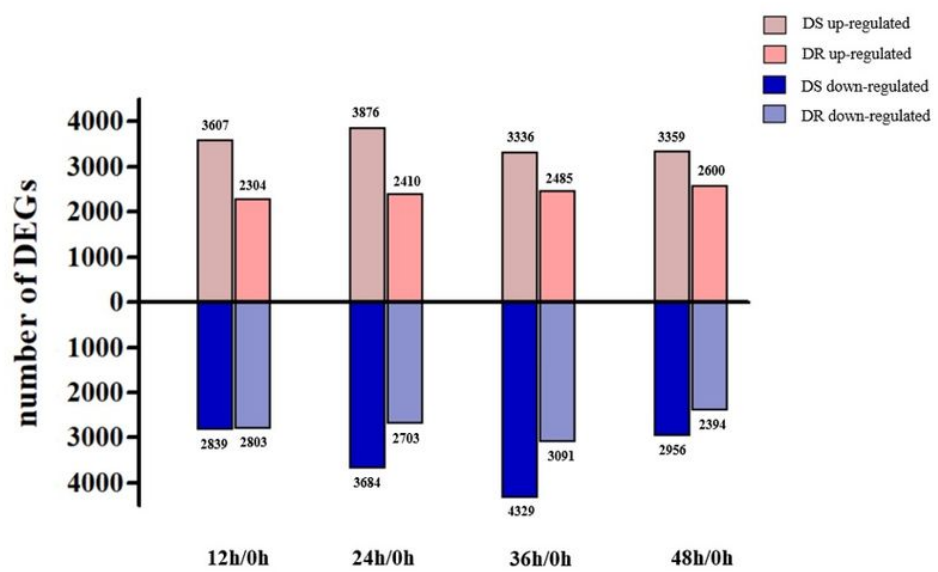

B

DS

DR
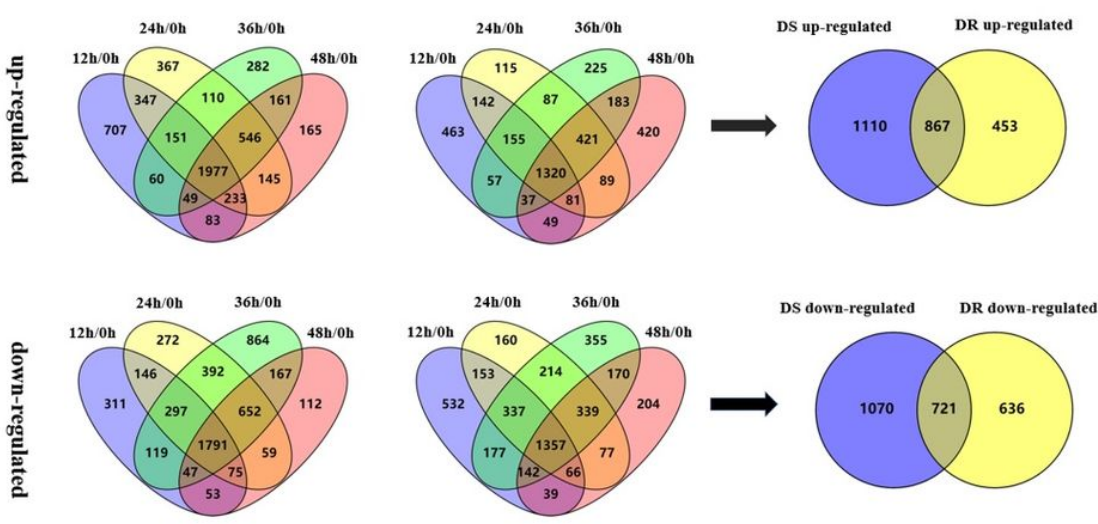

Figure 2

DEGs in DS and DR.
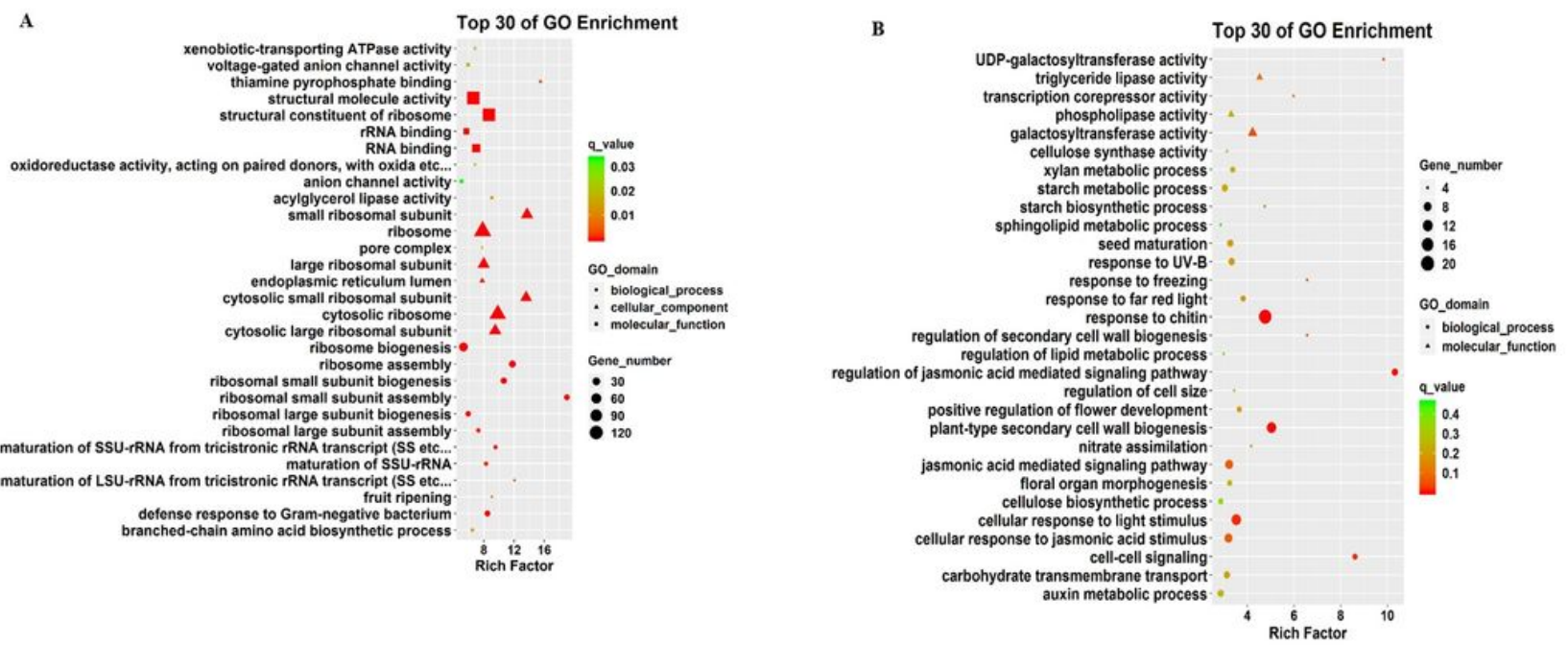

Figure 3 
Top 30 GO terms enriched function categories of co-up-regulated (A) and co-down-regulated (B) DEGs in two genotypes.
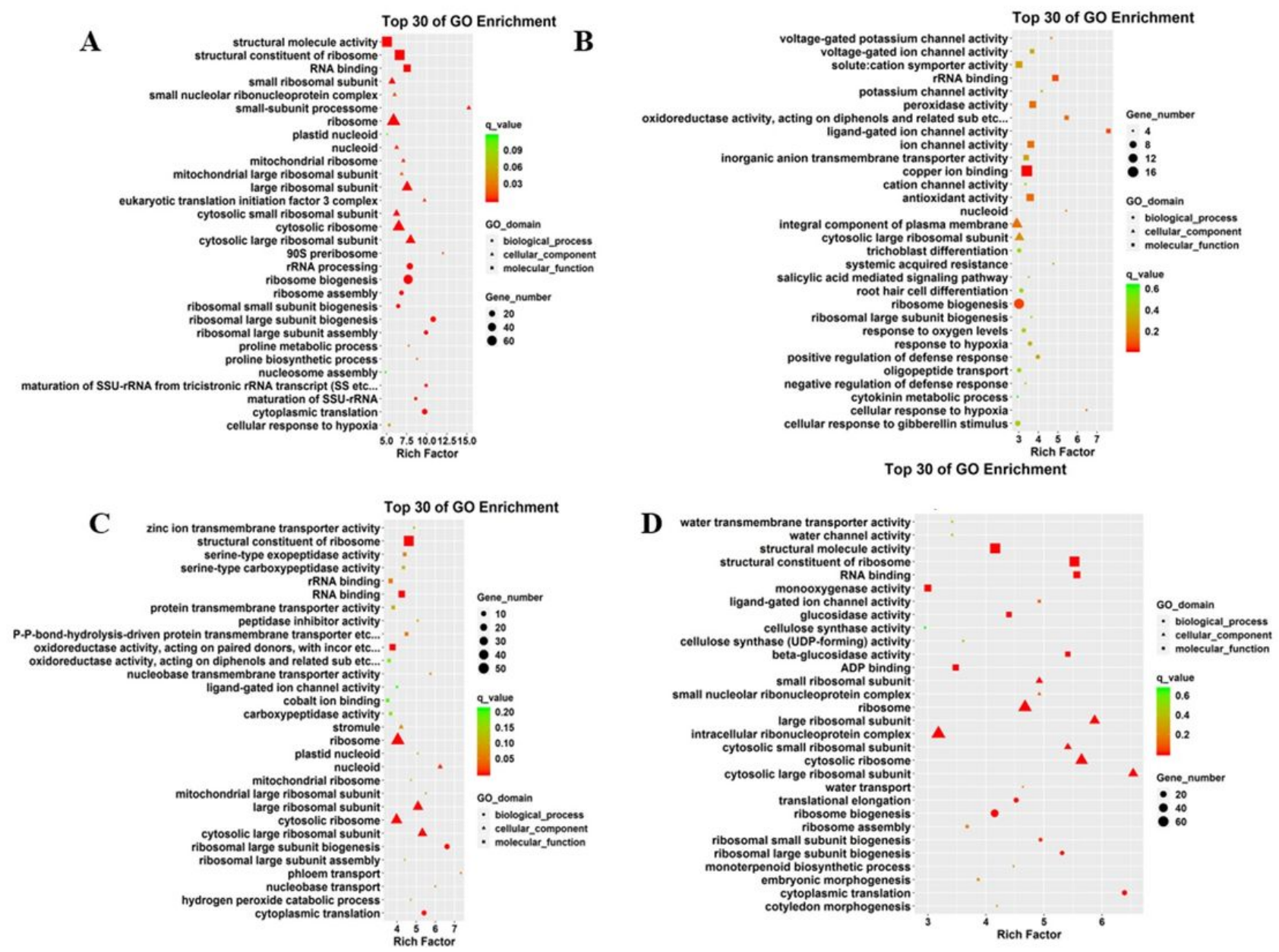

\section{Figure 4}

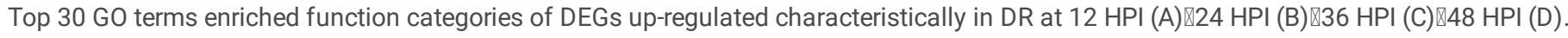




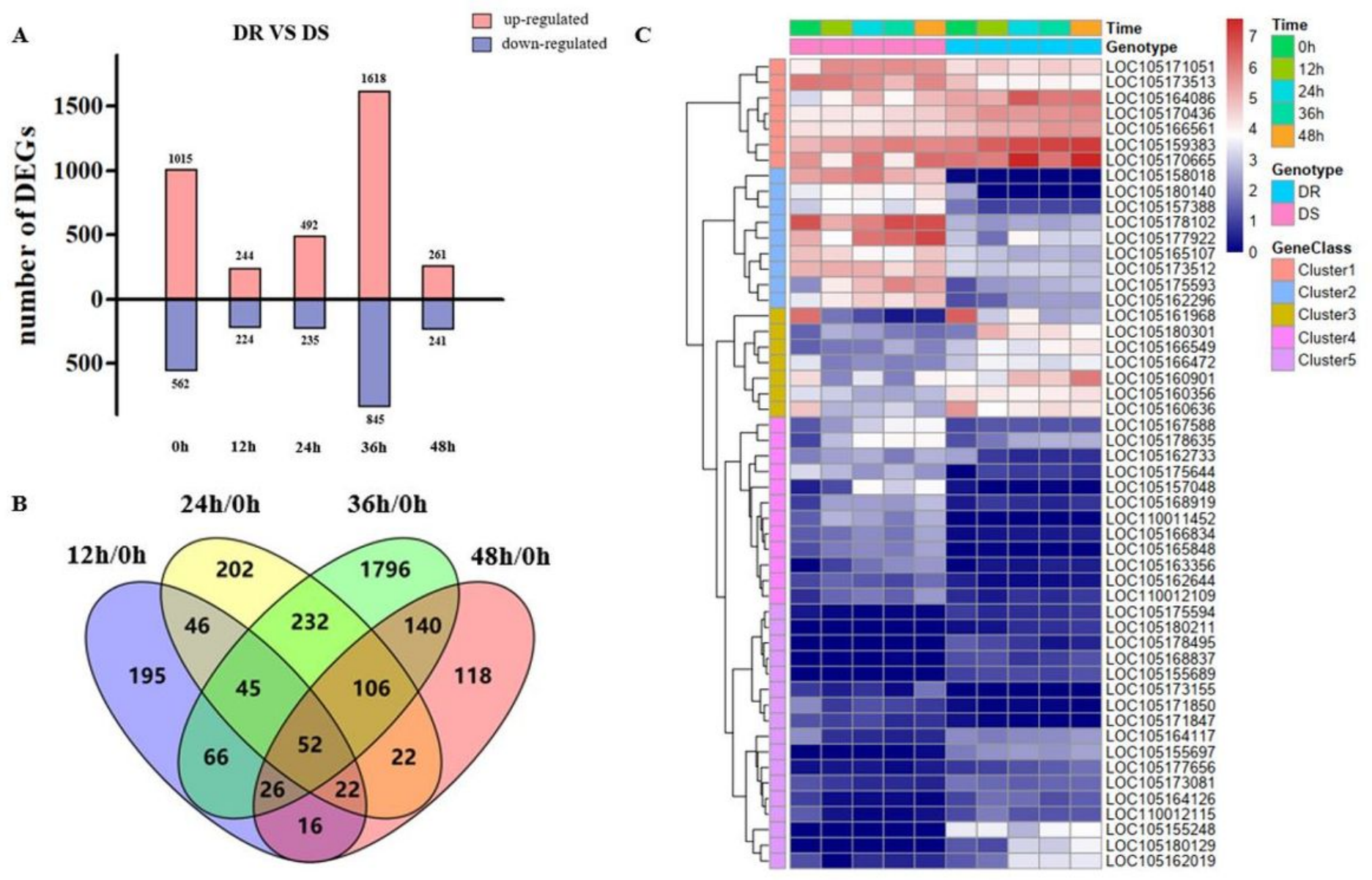

Figure 5

DEGs compared between DR and DS post-innoculation. A. Numbers of DEGs between DR and DS during MP stress. B. Common and unique DEGs between DR and DS post-innoculation. C. Expression patterns of 52 common DEGs between DR and DS post-innoculation. The value of gene expression is shown as $\log 2(\mathrm{FPKM}+1)$. 


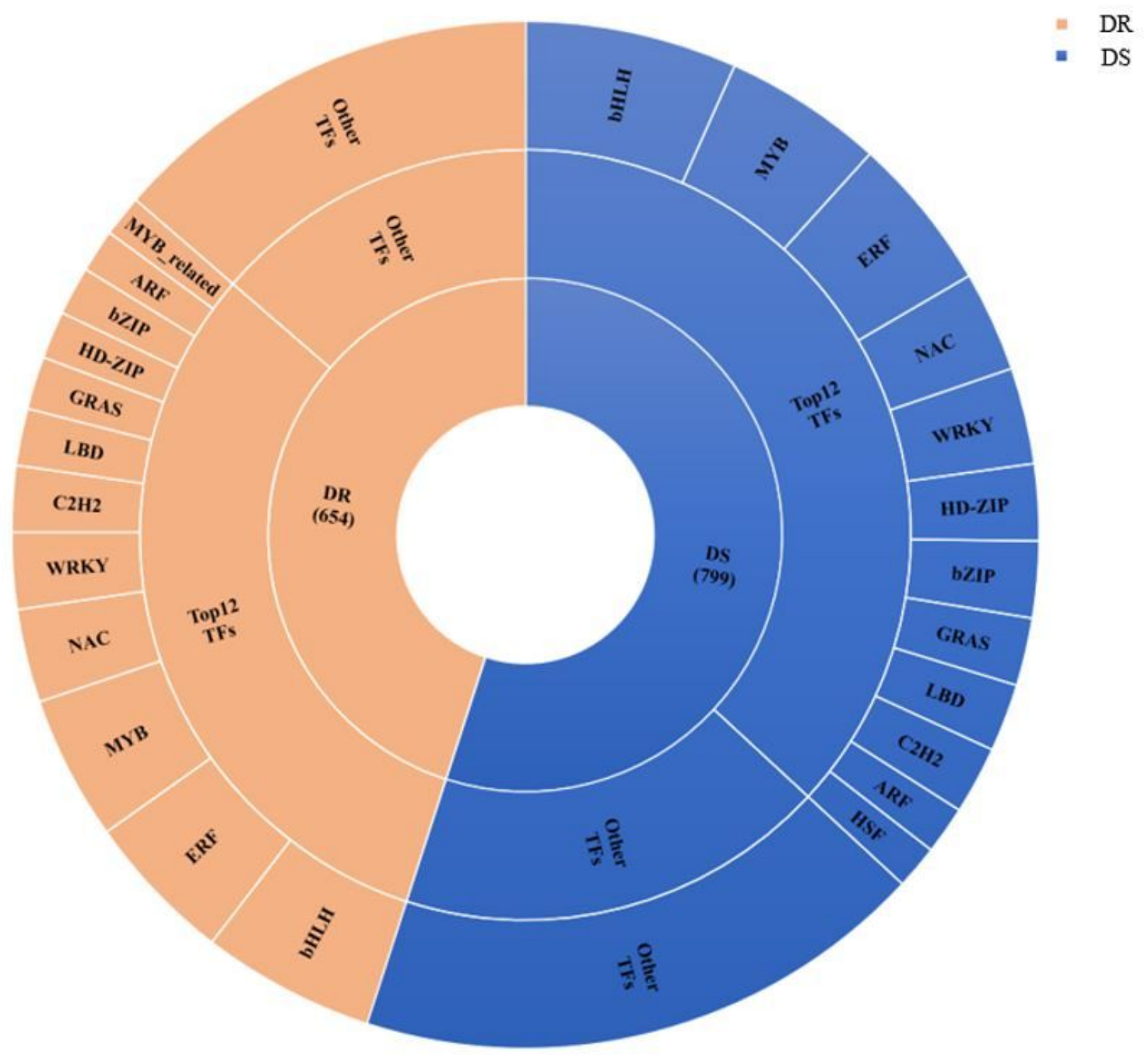

Figure 6

Overall TFs in DR and DS post-innoculation. 
A
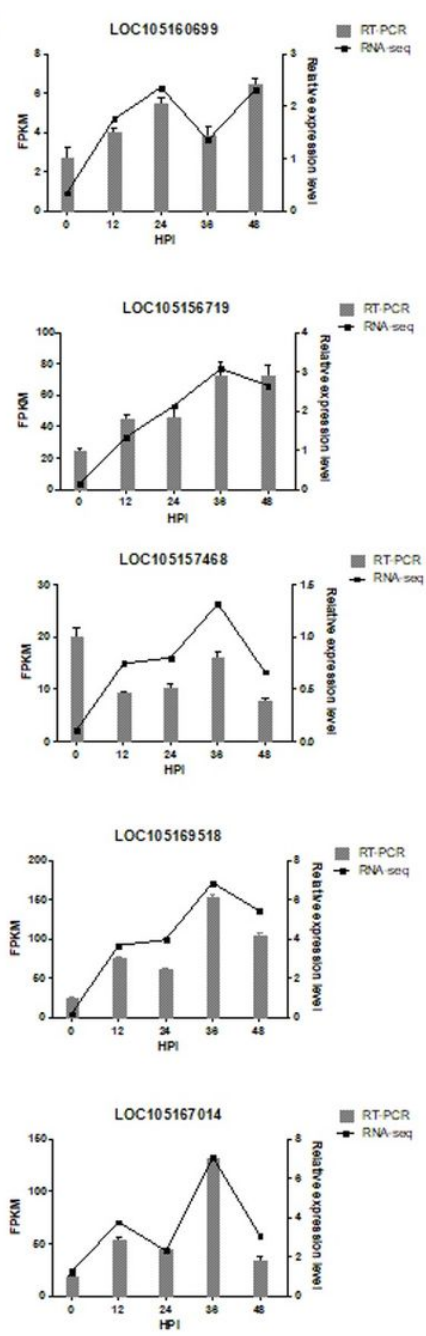

B
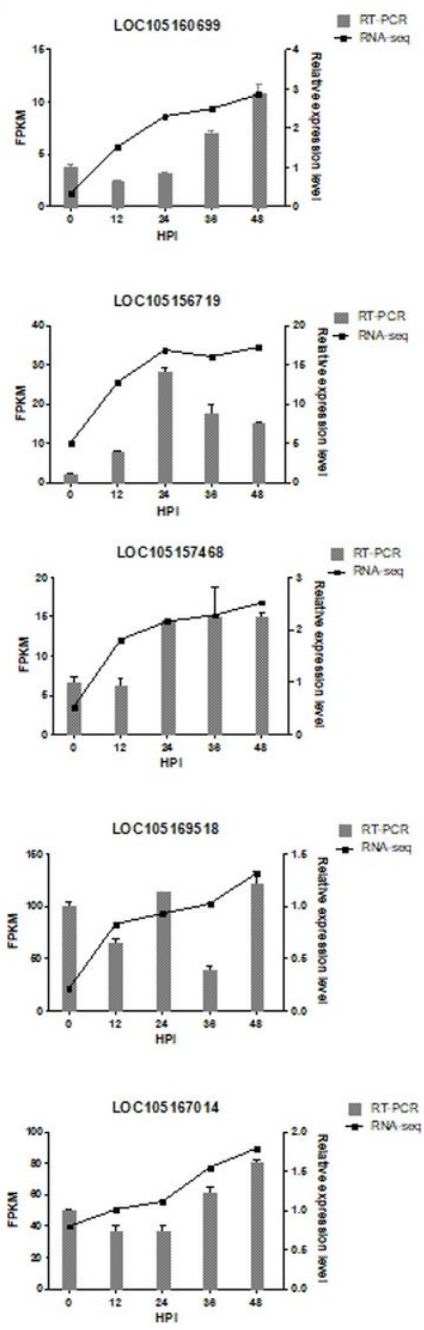

Figure 7

Quantitive RT-PCR validation of genes in DS (A) and DR (B). 


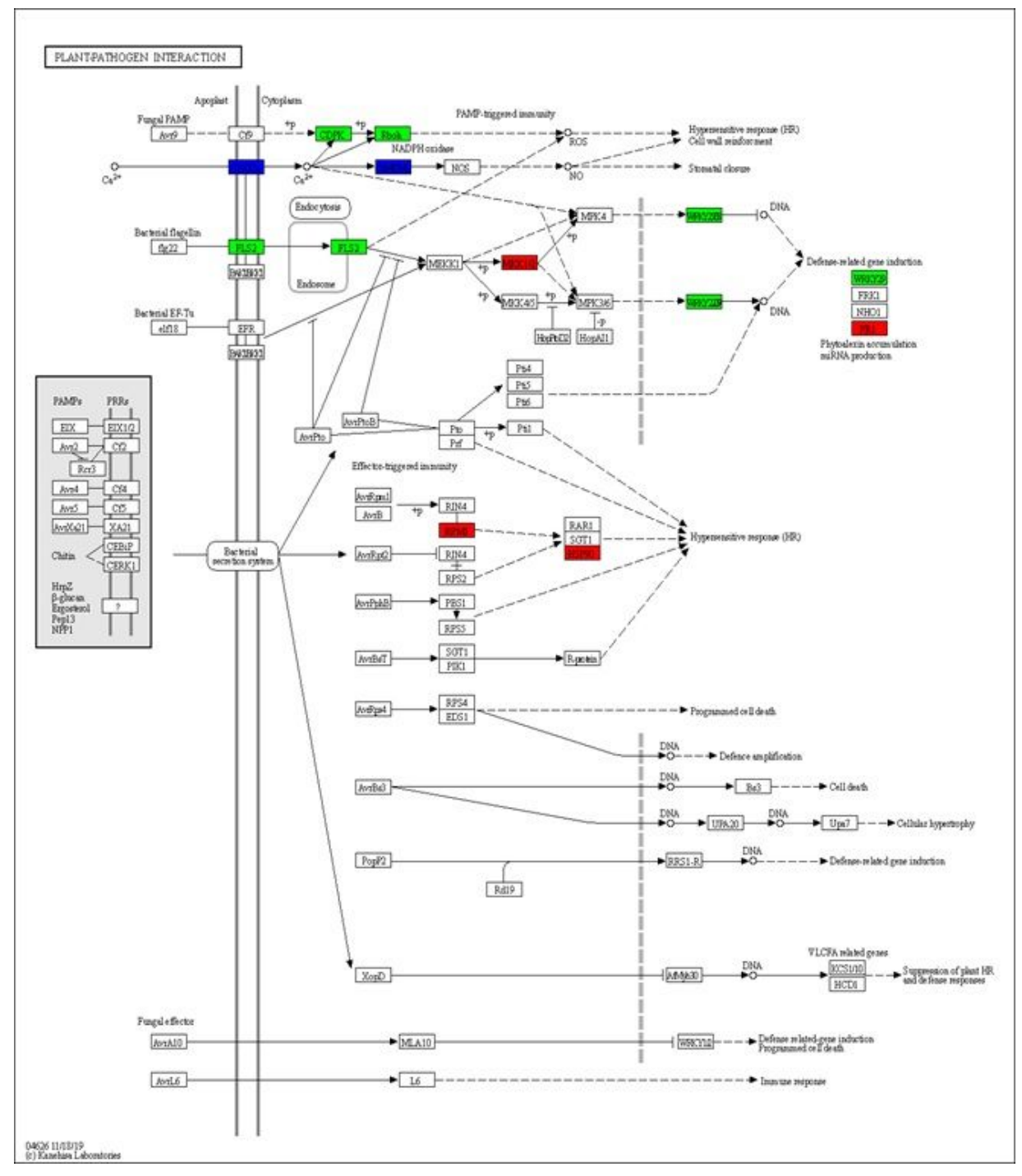

Figure 8

Plant-pathogen interaction map in DR. DEGs up-regulated is highlighted in red. DEGs down-regulated is highlighted in green. 


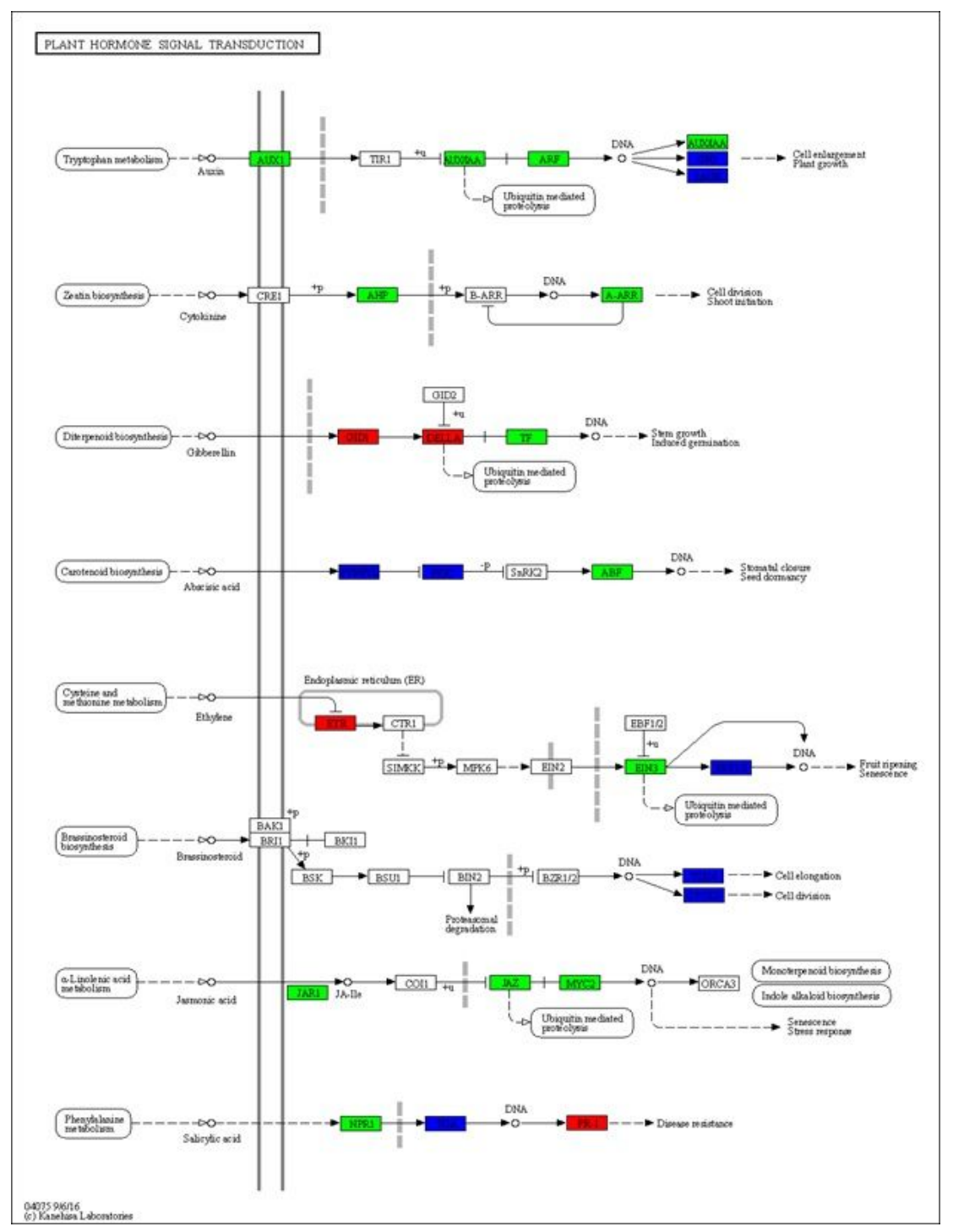

Figure 9

Plant hormone signal transduction map in DR. DEGs up-regulated is highlighted in red. DEGs down-regulated is highlighted in green.

\section{Supplementary Files}

This is a list of supplementary files associated with this preprint. Click to download.

- AdditionalfileFigureS1.docx

- AdditionalfileFigures2.docx

- AdditionalfileFigures3.docx

- AdditionalfileFigureS4.docx

- AdditionalfileTableS1.xlsx

- AdditionalfileTables2.xlsx

- AdditionalfileTables3.xIsx

- AdditionalfileTableS4.xlsx

- AdditionalfileTables5.xlsx

- AdditionalfileTableS6.xIsx

- AdditionalfileTables7.xIsx

- AdditionalfileTables8.xlsx 